\title{
Inventaire forestier : utilisation d'un compas forestier électronique
}

\author{
R. ZARNOVICAN et D. OUELLET \\ Service canadien des forêts, Centre de foresterie des Laurentides, \\ 1055, rue du P.E.P.S., C.P. 3800, Sainte-Foy (Québec), Canada G1V 4C7
}

\author{
Summary \\ Forest inventory with an electronic caliper
}

An electronic caliper with an optical encoder was developped for forest research studies. Connected to TRS- $80^{\oplus}$, model 100 portable computer, by "BCD" interface, this caliper permits the computerized errorfree dbh-date collection and storage in tandom access memory $(32 \mathrm{~K})$, by pressing of a simple button. Information such as species height and defects can be manually entered on the keyboard. The data can be reviewed, edited and analysed by application BASIC programs directly in the field, eliminating manual data transcription.

Key words : Electronic caliper, forest inventory, computerized data collection.

\section{Résumé}

Un compas électronique, comportant un encodeur optique a été développé. Relié à un microordinateur portatif par un interface, ce compas permet simultanément la saisie et l'informatisation du diamètre de l'arbre, et d'éliminer ainsi les erreurs associées à ces opérations.

Mots clés : Compas électronique, inventaire forestier, saisie informatisée des données.

\section{Problématique}

Traditionnellement, la saisie de données dendrométriques en forêt s'est effectuée par l'inscription sur les formulaires, et au bureau par l'informatisation. Ces deux étapes, nécessaires jusqu'à présent, comportent de sérieux inconvénients. En effet, il y a possibilité d'erreurs lors de la transcription des données au formulaire, car la personne qui reporte les données n'est habituellement pas celle qui prend la mesure. De plus, l'informatisation des données constitue aussi une source appréciable d'erreurs. 


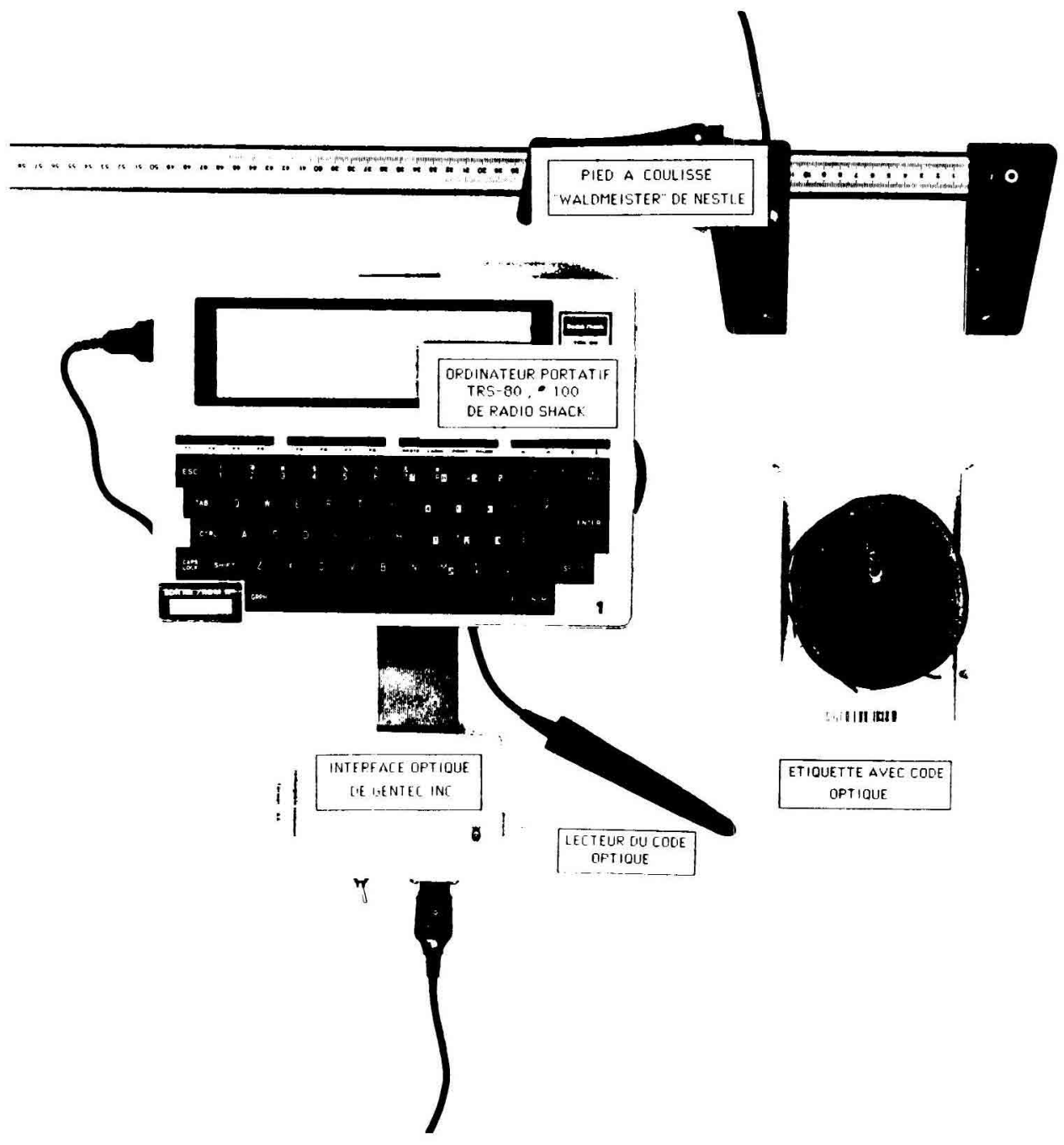

Compas electronique avec le micro-ordinateur portatif TRS-80, modèle 100 de Radio Shack. Electronic caliper and Radio Shack TRS-80, model 100 portable computer. 
L'utilisation d'un micro-ordinateur en forêt présente un intérêt majeur. En effet, cela permet d'éliminer les erreurs d'informatisation, puisque le transfert des données s'effectue par une communication directe entre deux ordinateurs. Toutefois, l'erreur de transcription persiste, qu'elle soit faite au fichier ou sur le formulaire. Aussi, avonsnous entrepris de développer un compas forestier électronique (photo 1). L'appareil doit permettre la prise de mesure et, par la simple pression d'un bouton, l'inscription de la mesure au fichier. Le compas forestier électronique a été développé en collaboration avec la firme Gentec Inc. de Sainte-Foy.

\section{Caractéristiques et fonctionnement}

Un compas forestier allemand, produit de Gottlieb Nestle Gimbh, a été retenu. II est robuste et maniable. La tige, d'une longueur de $60 \mathrm{~cm}$, d'une forme ovale et de profil creux, est fabriquée d'un alliage spécial ayant une bonne résistance à la flexion. Elle est protégée contre l'usure de frottement par une oxydation anodique. Le coulisseau et les équerres sont en polyamide renforcés de fibres de verre.

L'interface est formée de l'encodeur optique (fixé sur le coulisseau du compas) et des compteurs électroniques situés dans le boîtier de l'interface avec sa source d'alimentation. Fabriquée par Gentec Inc., l'interface a pour rôle de saisir le déplacement du coulisseau correspondant au diamètre de l'arbre. La lecture est effectuée au moyen d'une bande millimétrique (située sur la face inférieure du compas) par les deux diodes de l'encodeur qui acheminent des pulsations électriques aux trois compteurs électroniques. L'information est codée en « $\mathrm{BCD}$ » et acheminée, par câble plat, à la mémoire du micro-ordinateur qui constamment affiche l'ouverture du compas. Le sens du déplacement du coulisseau est transmis aux compteurs par l'activation première de l'une ou de l'autre des diodes. La saisie du diamètre de l'arbre et sa mise en mémoire s'effectue à l'aide du bouton d'acquisition, situé sur le boîtier de l'encodeur optique, tout comme le bouton de remise à zéro.

Le micro-ordinateur utilisé est le TRS- $80^{\text {(i) }}$ modèle 100 de Radio Shack, et sa mémoire vive est de $32 \mathrm{~K}$. La mise en mémoire des données s'effectue séquentiellement (ASCII) grâce à un programme conversationnel écrit en BASIC. Ce micro-ordinateur est équipé d'un interface de lecture de codes à barres, ce qui permet l'utilisation d'étiquettes à codes optiques pour l'identification des arbres des placettes permanentes.

Cet équipement électronique, en plus d'assurer l'enregistrement du diamètre dans la mémoire de l'ordinateur, permet également à l'utilisateur l'entrée au clavier de variables comme les caractéristiques stationnelles de la placette ou d'autres variables dendrométriques. A la fin d'une séance de travail, l'utilisateur peut transférer les données sur cassettes, sur disquettes ou à un autre ordinateur. Il peut ainsi libérer la mémoire du micro-ordinateur pour la prochaine séance de travail. En plus de servir au stockage des données, le micro-ordinateur peut évidemment servir de centre de calcul, et au traitement de texte. Ces avantages deviennent particulièrement intéressants pour certaines applications, notamment pour le secteur de la forêt privée. 


\section{Fiche technique}

Poids:

Compas électronique $(0,890 \mathrm{~kg})$;

Interface $(0,270 \mathrm{~kg})$;

TRS-80 $(1,775 \mathrm{~kg})$.

\section{Alimentation :}

Interface : 4 piles de type * AA * rechargeables nickel cadmium;

Micro-ordinateur : 4 piles de type « $\mathrm{AA}$ » rechargeables nickel cadmium.

Autonomie :

Interface : Jusquà 5 heures selon la température et l'humidité de l'air ;

Micro-ordinateur: Jusqu'à 20 heures, mais considérablement réduite pour une température ambiante inférieure à $-5^{\circ} \mathrm{C}$. L'usage d'un recoưrement protecteur plastifié contre l'humidité et la poussière est également nécessaîre.

Recu le 4 janvier 1988.

Accepté le 12 avril 1988. 